\title{
Aprendizagem simulada na nuvem
}

\section{Rafaela R. Jardim¹, Roseclea Medina ${ }^{1}$, Giliane Bernardi' ${ }^{1}$, Fabricio Herpich ${ }^{2}$, Andressa Facalde', Eduardo Lemos ${ }^{1}$}

\author{
1Universidade Federal de Santa Maria (UFSM) \\ Caixa Postal 5082 - 97.105-900 - Santa Maria - RS - Brazil \\ ${ }^{2}$ Universidade Federal do Rio Grande do Sul (UFRGS) \\ Caixa Postal 15.064 - 91.501-970 - Porto Alegre - RS - Brazil \\ \{rafa.rjardim, roseclea.medina, bgiliane, fabricio.herpich, \\ andressafacalde, elemos 04$\}$ @gmail.com
}

\begin{abstract}
Cloud Computing solutions can be used to support learning in $U$ learning environments and are an attractive alternative to maximize / flexible computing resources. Thus, this study aims to present a ubiquitous virtual laboratory U-Lab Cloud, which was associated with the technology of Cloud Computing and the U-learning features. a simulated case study, it was possible to check the evolution of the learning styles of students in a fictional educational process was carried out. With the results obtained, it was possible to demonstrate the feasibility of adopting cloud computing technology in educational settings.
\end{abstract}

Resumo. As soluções de Cloud Computing podem ser utilizadas para apoiar a aprendizagem em ambientes $U$-learning e são uma alternativa atrativa para maximizar/flexibilizar os recursos computacionais. Dessa forma, este trabalho objetiva a apresentação do laboratório virtual U-Lab Cloud, ao qual foi associado à tecnologia de Cloud Computing e as características de Ulearning. Foi realizado um estudo de caso simulado, onde foi possível verificar a evolução dos estilos de aprendizagem de estudantes fictícios em um processo educacional. Com os resultados obtidos, foi possível demonstrar a viabilidade da adoção da tecnologia de Cloud Computing em ambientes educacionais.

\section{Introdução}

A evolução das Redes de Computadores influenciou tanto em tamanho como em complexidade de infraestrutura. Para Filippetti (2008), esses avanços fizeram crescer a demanda e a exigência por profissionais melhor qualificados, capazes de suportar todas as nuances tecnológicas desses ambientes. Com isso, evidencia-se a importância da formação de profissionais aptos a planejar e desenvolver atividades técnicas na área de Redes de Computadores. Dessa forma, as aulas práticas possibilitam a experimentação dos conceitos vistos pelos estudantes. Dado tal cenário, torna-se necessário buscar novas formas e estratégias para o ensino de Redes de Computadores contemplando a utilização dos recursos tecnológicos disponíveis atualmente.

Diante de diversas ferramentas que apresentam grande potencial educacional, tem-se os ambientes virtuais imersivos ou também chamados mundos virtuais, que permitem experiências de simulação e atividades de colaboração. O paradigma educacional imersivo tem como objetivo disponibilizar espaços tridimensionais onde o estudante pode transitar e vivenciar experiências em um ambiente altamente interativo (ORGAZ et al. 2012). Neste cenário, surge a necessidade de propiciar ambientes virtuais que forneçam recursos computacionais adaptados às particularidades de cada estudante.

Para a adaptação e personalização de ambientes educacionais podem ser 
V Congresso Brasileiro de Informática na Educação (CBIE 2016)

Anais do XXVII Simpósio Brasileiro de Informática na Educação (SBIE 2016)

utilizados elementos de ubiquitous learning (U-Learning). Yahya et al. (2010) definem $U$-Learning como um paradigma de aprendizagem localizado em ambientes computacionais ubíquos, que permite o estudante aprender, no lugar, no tempo e na direção correta. Existem diversos aspectos que devem ser introduzidos na projeção dos ambientes $U$-Learning, como as informações de contexto.

Conforme Dey (2001), o contexto é qualquer informação que possa ser usada para caracterizar a situação de entidades que sejam consideradas relevantes para a interação entre um usuário e uma aplicação. Herpich et al. (2016) corroboram afirmando que, a partir das informações contextuais coletadas, e.g. contexto computacional, físico e de tempo, diversas são as possibilidades de utilização das mesmas em aplicações computacionais, destacando-se por exemplo, o fornecimento de serviços personalizados aos usuários, tais como a adaptação de conteúdos e ferramentas conforme as preferências dos mesmos.

A tecnologia de Cloud Computing (Computação em Nuvem), quando utilizada no campo educacional, pode impactar positivamente na construção, manutenção e atualização de infraestruturas. As soluções de Cloud Computing podem ser utilizadas para apoiar a aprendizagem em ambientes $U$-learning, pois são alternativas para maximizar e flexibilizar os recursos computacionais.

$\mathrm{Na}$ presente pesquisa foi implementado um ambiente de nuvem privada para hospedar o laboratório virtual ubíquo U-Lab Cloud. Esse ambiente apresenta características de um sistema U-learning, sendo adaptável ao contexto dos estudantes e foi disponibilizado por meio da tecnologia de Cloud Computing. Desse modo, as informações de contexto consideradas nesse trabalho são: o estilo cognitivo, o tipo de dispositivo e a velocidade de rede.

Este artigo está organizado da seguinte forma: na seção II são explanados os conceitos relacionados a Cloud Computing e a U-learning; a seção III descreve a metodologia utilizada nesta pesquisa; a seção IV apresenta o ambiente desenvolvido; na seção V encontra-se a implementação realizada; já a análise dos resultados encontra-se na seção VI; por fim, na seção VII estão as considerações finais deste trabalho.

\section{Referencial Teórico}

A fim de contribuir nas atividades realizadas durante esta pesquisa, foi desenvolvida uma revisão bibliográfica sobre os temas relevantes para o estudo. As seções a seguir apresentam uma visão geral dos conceitos sobre Cloud Computing e U-learning.

\subsection{Cloud Computing}

Na literatura encontram-se diversas definições para Cloud Computing como pode ser visto em (Caminero et al. 2013; Wang et al. 2010), sendo a mais apropriada para este trabalho a definição cunhada por Mell e Grance (2011), onde Cloud Computing é definida como um modelo computacional que permite o acesso sob demanda da rede a um pool de recursos computacionais configuráveis que podem ser liberados com um esforço mínimo de gerenciamento.

A utilização da Cloud Computing pode auxiliar os usuários a se libertarem das amarras da tecnologia e contribuir para a construção da sua aprendizagem (BAI, 2011), uma vez que toda a aplicação encontra-se disponível na "nuvem". Assim, para usufruir da sua aplicação, os usuários precisam apenas de um navegador simples com conectividade á Internet (JADEJA e MODI, 2012). Além disso, outra vantagem para o usuário desse tipo de aplicação é que a mesma dispensa atualização de hardware e software. 
V Congresso Brasileiro de Informática na Educação (CBIE 2016)

Anais do XXVII Simpósio Brasileiro de Informática na Educação (SBIE 2016)

\subsection{U-learning}

A aprendizagem ubíqua, também conhecida como U-learning foi impulsionada pelo avanço da tecnologia e da comunicação de rede (JEONG e YI, 2014). Conforme Hooft (2006) a computação ubíqua quando relacionada à educação abrange os participantes ativos no processo de ensino-aprendizagem, pois possibilita que professores e alunos recebam as informações e criem conhecimentos novos de forma individual ou colaborativa. Para Rabello (2012), o paradigma da computação ubíqua promove a ideia da troca de informações a qualquer hora e em qualquer lugar, por meio da utilização, de forma transparente, inteligente e integrada às tecnologias computacionais. Hooft (2006) complementa que a computação ubíqua quando relacionada à educação abrange os participantes ativos no processo de ensino-aprendizagem, pois possibilita que professores e alunos recebam a informação e criem conhecimentos novos de forma individual ou colaborativa.

Desta forma, diversas áreas de pesquisa que integram $U$-learning, entre elas pode-se citar a computação móvel e a ciência de contexto. O laboratório virtual é um ambiente que aborda características de U-learning, visto que realiza adaptações baseadas no contexto do usuário e no computacional. Para identificar as informações que interferem na sua utilização e realizar as adaptações necessárias nesse ambiente, foi-lhe integrado o Módulo Ảdaptativo. Essa integração foi realizada com intuito de evitar problemas abruptos relacionados com a baixa velocidade de conexão ou com os tipos de dispositivos, uma vez que, muitas vezes essas limitações limitam ou até impedem o estudante de carregar e visualizar os materiais corretamente. Esses entraves não são mais admitidos pelos usuários. Por fim, o estudo realizado nesta seção embasou o desenvolvimento deste trabalho, no qual procurou-se identificar e atender as características de ubiquidade apresentadas acima.

\section{Metodologia}

O objetivo principal desta pesquisa foi criar um ambiente de nuvem privada com características de um sistema U-learning e que, integrado ao Módulo Adaptativo, auxilie/facilite a aprendizagem de Redes de Computadores.

Para o desenvolvimento deste trabalho, foi empregada a pesquisa experimental, a qual tem por finalidade selecionar variáveis capazes de modificar o ambiente de estudo. Tendo em vista a natureza da pesquisa, esta é classificada como uma pesquisa aplicada, cujo propósito é buscar a solução de um objetivo específico. Primeiramente, foi definido o projeto de arquitetura, que contempla a infraestrutura de hardware necessária para o desenvolvimento desta pesquisa. A infraestrutura utilizada para realizar o experimento é composta por três máquinas, sendo: um servidor Dell Power Edge T300 com processador Intel Xeon Quadcore X3363 $2.83 \mathrm{GHz}$ com 4 núcleos físicos e 4 núcleos virtuais, memória RAM de $8 \mathrm{~GB}, 2$ discos rígidos de 500GB e dois servidores com processador Intel Core2 Quad 2.88GHz, contendo 4 núcleos físicovirtuais, memória RAM de $8 \mathrm{~GB}$ e disco rígido de 500GB.

A segunda etapa considera o problema, os objetivos e a contribuição pretendida com esta pesquisa. Nesta fase, foi definida e avaliada a plataforma de Cloud Computing OpenNebula. A linguagem de programação selecionada para a implementação do Módulo Adaptativo foi o PHP, por ser a linguagem nativa do AVA Moodle, e, para o desenvolvimento do medidor de velocidade de rede foi utilizada a liguaguem JavaScript. A terceira fase consistiu na instalação das ferramentas que compõem o laboratório virtual U-Lab Cloud, sendo elas: WampServer4 versão 2.2 (inclui as ferramentas: Apache 2.2.22; Mysql 5.5.24; PHP 5.3.13 e PhpMyadmin 3.4.10.1); Open Simulator versão 0.7.4; Moodle 6 versão 2.5; Sloodle 7 versão 2.1 for Moodle 2.5.

$\mathrm{Na}$ quarta etapa, foi realizada a inserção do Módulo Adaptativo. Esse Módulo tem por finalidade coletar e tratar as informações, como a velocidade de rede, tipo de 
V Congresso Brasileiro de Informática na Educação (CBIE 2016)

Anais do XXVII Simpósio Brasileiro de Informática na Educação (SBIE 2016)

dispositivo e o estilo cognitivo dos alunos. Na quinta etapa, foi definido o método de avaliação do U-Lab Cloud.

Para orientar a seleção dos métodos de avaliação utilizados nesta pesquisa, foi realizado um mapeamento sistemático sobre as avaliações de implementações de Cloud Computing para Ambientes Educacionais. Com base nos resultados obtidos, foi possível concluir que esse tema ainda é pouco explorado e que não existe um método bem definido para avaliação de sistemas educacionais disponibilizados em ambiente de nuvem.

Considerando o referido cenário, este trabalho adotou como método de avaliação as técnicas de simulação computacional, em razão de que a avaliação com estudantes reais necessita de um período grande de tempo e um número considerável de estudantes dispostos a colaborar. Conforme Iglesias et al. (2009), quando se trata de sistemas em avaliação é difícil de convencer uma quantia suficiente de estudantes a participar desse tipo de experimento. Para isso, foi necessário realizar uma adaptação na ferramenta de simulação MADEA (Modelagem Automática e Dinâmica de Estilos de Aprendizagem), a qual foi desenvolvida por Dorça (2012) em sua tese. Essa ferramenta tem por objetivo detectar e corrigir de forma dinâmica e automática as inconsistências de estilos de aprendizagem do estudante.

\section{Laboratório Virtual: U-Lab Cloud}

Para disponibilizar o U-Lab Cloud foi utilizada a Cloud Computing com o propósito de agregar a característica de elasticidade, facilitando o gerenciamento dos recursos computacionais de cada máquina virtual criada na nuvem privada, sendo possível gerenciar de forma dinâmica o funcionamento das máquinas virtuais de acordo com a necessidade de cada usuário. A outra contribuição pretendida com esta pesquisa é empregar as características da Computação Ubíqua ao laboratório virtual disponibilizado no ambiente de nuvem. Com isso, pretende-se auxiliar os estudantes a receber a informação adequada e no momento correto.

Nesse ambiente, a apresentação do material disponibilizado ao aluno receberá a adaptação de acordo com o perfil cognitivo, a velocidade de conexão e o tipo de dispositivo. Essas informações serão identificadas por meio do Módulo Adaptativo que está integrado ao AVA Moodle. O Módulo Adaptativo tornará o U-Lab Cloud dinâmico, uma vez que auxilia na identificação de variáveis de contexto e atende ás características de mobilidade e disponibilidade.

A arquitetura proposta para o laboratório virtual foi projetada em três camadas: Apresentação, Aplicação e Dados (Figura 1). A camada de Apresentação é a mais superficial deste ambiente e fornece a interface do ambiente. Para acessá-la, o usuário precisará ter instalado em seu dispositivo/computador apenas um browser, que permita a navegação na Internet para ter acesso ao laboratório virtual U-Lab Cloud.

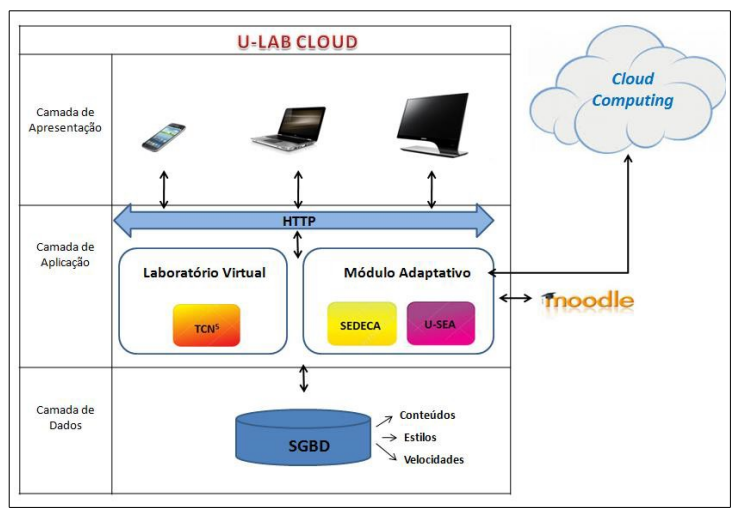

Figura 1. Arquitetura do U-Lab Cloud 
A camada de Aplicação é a intermediária, sendo os seus principais componentes o $\mathrm{TCN}^{5}$ (Voss, 2014), o SEDECA (Mozzaquatro, 2010) e o U-SEA (Piovesan, 2011). Nesta camada, o laboratório virtual é composto pelas ferramentas OpenSim e Sloodle utilizadas no desenvolvimento do TCN ${ }^{5}$. Também encontra-se o Módulo Adaptativo, o qual foi uma integração do SEDECA e do U-SEA e tem por objetivo principal adaptar o ambiente de acordo com as características de contexto do estudante, e.g. a velocidade de conexão e o estilo cognitivo.

O Módulo Adaptativo irá identificar essas informações por meio do SEDECA e do U-SEA, que foram integrados à plataforma Moodle. A identificação do estilo cognitivo será realizada pelo SEDECA, onde o usuário responderá um questionário para definir suas preferências em relação ao tipo de apresentação do conteúdo por exemplo, vídeo ou texto. Já o U-SEA irá verificar a velocidade de conexão e adaptará o ambiente considerando o tipo de material que é suportado com a velocidade de conexão identificada.

Por fim, o Módulo Adaptativo incorporado na Moodle se comunicará com o SGBD (Sistema Gerenciador de Banco de Dados) armazenado na camada Dados e adaptará a apresentação do conteúdo no laboratório virtual conforme com a preferência de cada estudante.

Ao contrário dos ambientes tradicionais, este dispensará a instalação, a manutenção ou a atualização de programas, pois estará sendo executado na nuvem de forma transparente, ou seja, não apresentará nenhuma modificação aparente para o aluno, atendendo às características de um ambiente U-learning. A introdução dos elementos de U-learning como a mobilidade, possibilita o acesso ao ambiente educacional de forma mais eficiente para alunos e professores.

\section{Implementacão}

A implementação do U-Lab Cloud foi baseada no desenvolvimento de um ambiente de nuvem privada. Para isso, primeiramente foi necessário definir a plataforma de Cloud Computing mais adequada a esta pesquisa.

Assim sendo, dois aspectos principais foram considerados: o modelo de serviço e a infraestrutura. O laboratório virtual pertence ao modelo de serviço Infraestrutura como Serviço (IaaS), uma vez que fornece a infraestrutura para desenvolvimento de atividades de práticas do ensino de Redes de Computadores.

Assim, foi pesquisada, projetada e configurada a infraestrutura física para realização do experimento. $\mathrm{O}$ ambiente de testes utilizado é composto por três máquinas: na máquina principal foram atribuídas as funções de cloud controle (CC), cluster controle (CLC) e storage controller (SC); as outras duas máquinas receberam a função de node controller, as quais fornecem o processamento da nuvem.

Logo, foi implementada a plataforma de Cloud Computing OpenNebula. Para realizar a virtualização, foi selecionado o hipervisor VMware ESXi, por ser compatível com a plataforma de Cloud Computing selecionada. Com a virtualização é possível criar e administrar diversas máquinas virtuais no mesmo sistema operacional.

Assim, ao utilizar o recurso de virtualização garante-se níveis adequados de disponibilidade para o U-Lab Cloud, visto que caso a máquina virtual em que o ambiente estiver rodando falhe, o ambiente automaticamente será migrado para outra. Com isso, tem-se a intenção de manter o ambiente acessível, assegurando a sua disponibilidade. Dessa maneira, pode-se dizer que o laboratório virtual apresenta um dos elementos que compõe as características pretendidas para este ambiente de Cloud Computing. 
Para a utilização do laboratório virtual no ambiente de nuvem privada, foi instanciada no VMware uma máquina virtual com sistema operacional Windows. Nela foi realizada a instalação das ferramentas instaladas no ambiente de Cloud Computing para criação do U-Lab Cloud. Na sequência, foi implantada a plataforma Moodle v. 2.5, onde foi criada a disciplina Redes de Computadores e foram inseridos alguns materiais e.g slides e vídeos.

O próximo passo foi a construção da interface do ambiente virtual 3D. Para isso, foi instalada a ferramenta OpenSim. Com o OpenSim e o Moodle em funcionamento, foi necessário integrá-los por meio da ferramenta Sloodle. Para isso, foi necessário copiar as pastas dessa ferramenta para o diretório do Moodle. Os materiais disponibilizados no Moodle foram integrados com o laboratório virtual por meio das aplicações "quiz chair" e "presenter" disponibilizadas nesse aplicativo. Por fim, foi realizada a instalação do viewer Singularity, necessário para visualização da interface gráfica do U-Lab Cloud (Figura 2).

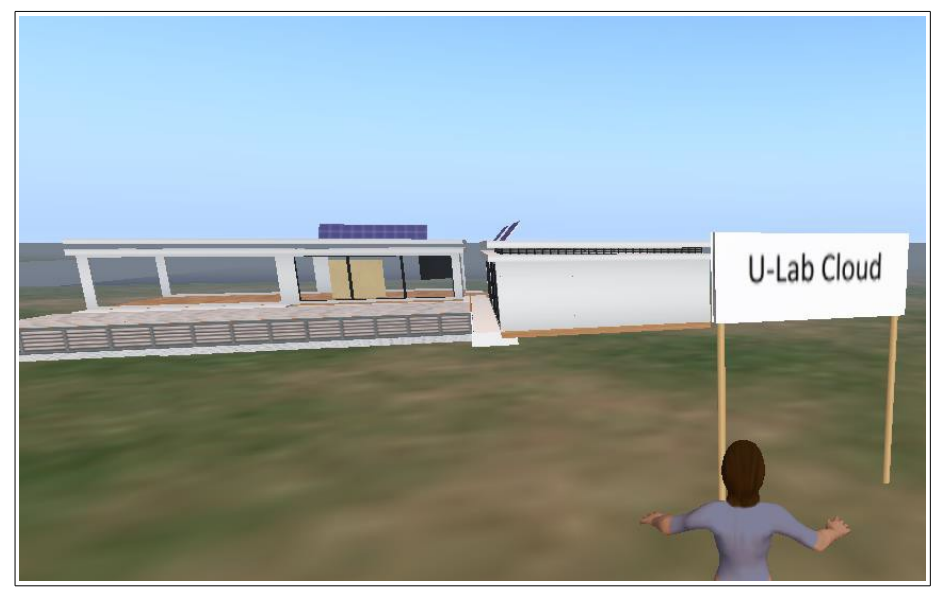

Figura 2. Ambiente U-Lab Cloud

Na figura 2, pode ser visualizado a interface gráfica do laboratório virtual no OpenSim. Dessa forma, o processo de implementação do U-Lab Cloud foi finalizado, onde pôde-se perceber diversas vantagens ao adotar a tecnologia de Cloud Computing em conjunto com as características U-learning. O baixo custo para o usuário é a principal delas, pois, para acessarem o laboratório virtual, não será necessário possuir tecnologia de ponta. Os usuários poderão acessar o ambiente de seus computadores pessoais (PCs), telefones celulares, tablets, mesmo que possuem uma configuração mínima, sendo necessário apenas que seus equipamentos estejam conectados a Internet.

Além disso, foi adaptada e integrada a plataforma Moodle a ferramenta de Modelagem de Estilos de Aprendizagem (MADEA) para realização dos testes. A principal finalidade de utilizar esta ferramenta de simulação computacional foi a possibilidade de obter uma visão geral sobre o funcionamento do ambiente, antes que o mesmo seja submetido a testes com estudantes reais.

\section{Análise dos Resultados}

O estudo de caso simulado foi baseado na disciplina de Redes de Computadores disponibilizada na plataforma Moodle. A disciplina utilizada neste estudo de caso é real, contudo, o acompanhamento e os estudantes foram simulados com o auxílio da ferramenta MADEA. Para empregá-la neste trabalho, foi necessário realizar a adaptação e a integração dela com a plataforma Moodle. 
Para demonstrar a integração das ferramentas mencionadas, foi definido um estudante fictício com a seguinte preferência: ativo (forte), sensitivo (forte), visual (moderada) e sequencial (fraca). Com os resultados obtidos nesta simulação, foi possível observar a atualização dos estilos de aprendizagem do estudante fictício no decorrer da disciplina de Redes de Computadores, com intervalos de 10 iterações. Esta simulação computacional considerou modelos probabilísticos, onde as probabilidades são determinadas pelo valor de cada preferência dos estilos de aprendizagem.

A Figura 3 mostra a dimensão de Processamento, que representa como o estudante com forte preferência pelo estilo Ativo procede em relação ao processamento de informação. Esse estudante com preferência por uma experimentação ativa, possui preferência por trabalhos em pequenos grupos e que envolvam discussão durante o andamento da disciplina.

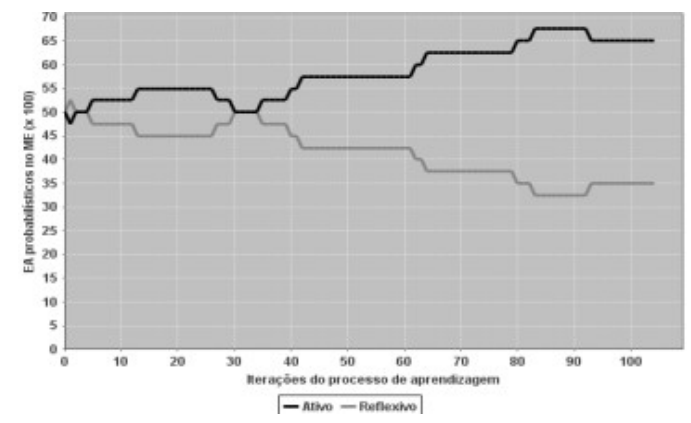

Figura 3 - Dimensão de Processamento.

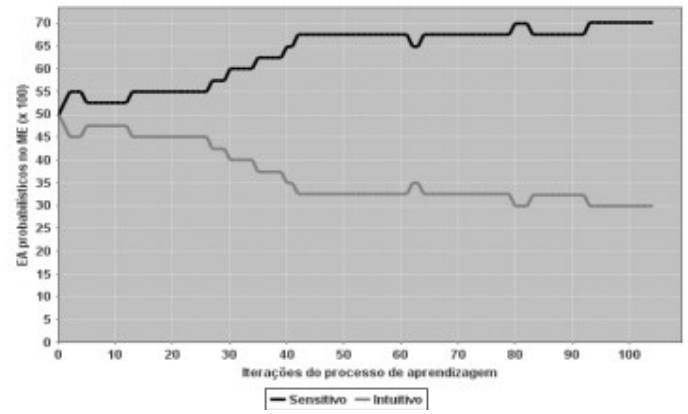

Figura 4 - Dimensão de Percepção.

Na Figura 3, foi possível observar como evoluiu o estilo de aprendizagem probabilístico do estudante com preferência pela experimentação ativa. Assim, percebese que a preferência pelo estilo Ativo (forte) foi mantida durante todo o processo de aprendizagem. Entretanto, observa-se que em dois curtos espaços de tempo por volta de (05 e 30 iterações) o estilo de aprendizagem apresentou-se inconsistente, mas foi corrigido a medida que a quantidade de iterações cresceu.

A atualização da dimensão Percepção pode ser visualizada na Figura 4. Essa dimensão diz respeito a qual tipo de informação é melhor percebida pelo estudante. $\mathrm{O}$ estudante com forte preferência pelo estilo Sensitivo está propenso a encontrar no ambiente educacional os exemplos do mundo real que disponibilizam datas, dados experimentais e informações que podem ser percebidas pelos seus sentidos (ver, ouvir).

$\mathrm{Na}$ Figura 4, ao analisar o progresso do estilo de aprendizagem Sensitivo (forte), pôde-se constatar que durante a trajetória tal estilo manteve-se consistente. Após um terço do caminho percorrido (por volta de 30 iterações), percebe-se indícios de que o estilo de aprendizagem tornou-se mais estável.

Na Figura 5, é demonstrada atualização da dimensão Entrada. Essa dimensão define qual o tipo de informação é efetivamente percebida pelo estudante que apresenta preferência fraca pelo estilo Visual. Esse estilo é mais propenso a percepção de figuras, diagramas, linhas de tempo, gráficos e filmes. 


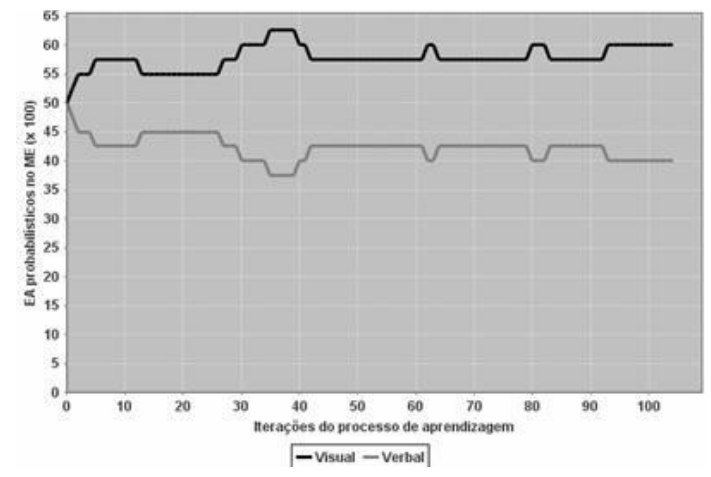

Figura 5 - Dimensão de Entrada.

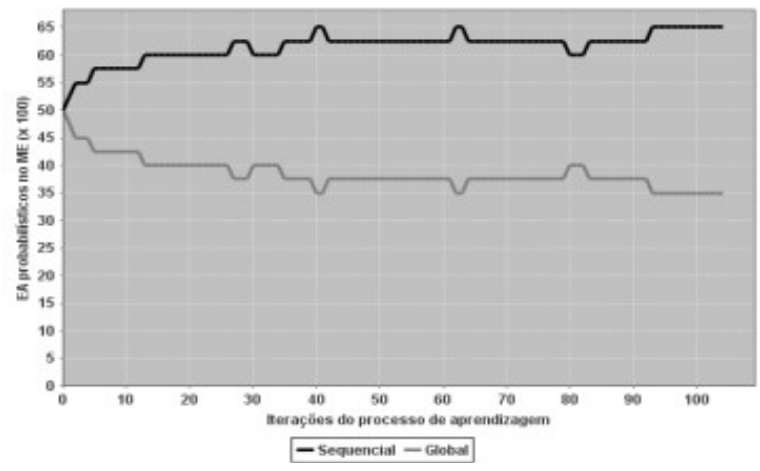

Figura 6 - Dimensão de Saída

A Figura 5 mostra os valores assumidos pelo estilo de aprendizagem Visual (fraco) durante o processo de aprendizagem simulado. Nota-se indícios de que o estilo de aprendizagem manteve-se instável até aproximadamente metade do processo por volta de 41 iterações). Após, percebe-se que o estilo tornou-se consistente.

Na Figura 6, é possível verificar a evolução da dimensão Organização. Essa dimensão determina como o estudante prefere progredir dentro da disciplina, sendo que neste caso, o estudante fictício apresenta fraca preferência pelo estilo de aprendizagem Sequencial. Dessa forma, ele está propenso a seguir uma progressão lógica e linear do conteúdo, ou seja, tem preferência por uma sequência de passos para solucionar problemas. A Figura 6 apresenta graficamente indícios de que durante o processo de aprendizagem, o estilo de aprendizagem mostrou-se bem definido, mesmo apresentando força de preferência fraca no modelo inicial de estudante.

O estudo de caso realizado demonstrou-se condizente com o objetivo desta abordagem, que buscou verificar o comportamento do estudante e do ambiente. A utilização de técnicas de simulação foi significativa para a avaliação deste trabalho, uma vez que propiciou executar testes em pouco tempo, o que seria dificultoso e mais complexo de realizar com estudantes reais. Dessa forma, acredita-se com os resultados desta avaliação, o U-Lab Cloud encontra-se apto para ser utilizado por estudantes reais em um processo de ensino-aprendizagem na disciplina de Redes de Computadores.

\section{Considerações Finais}

Com relação ao estudo de caso desenvolvido, foi possível concluir que a utilização de técnicas de simulação para avaliar o funcionamento de um sistema educacional antes de submetê-lo a testes com estudantes reais foi fundamental, devido às limitações encontradas nesta etapa. Pode-se destacar como a principal limitação evidenciada em processos de avaliação de ambientes educacionais, a fragilidade das respostas obtidas por meio de questionários. A utilização desse recurso por estudantes exige muito tempo e concentração, pois geralmente esse instrumento é extenso e os estudantes não estão a par da importância das suas respostas. Por isso, os estudantes acabam respondendo sem ter o cuidado de analisar o impacto do seu feedback, o que pode tornar os resultados pouco relevantes, até mesmo incompatíveis com os dados reais dos estudantes.

Além disso, outra limitação percebida foi a dificuldade de disponibilização de horários de aula em cursos reais, visto que os professores seguem suas atividades conforme seus planejamentos, não sendo possível realizar adaptações, uma vez que, trabalham com um calendário acadêmico pouco flexível. E, ainda, existe a preocupação com a exposição de informações de alunos e professores nas instituições de ensino.

Neste cenário, a avaliação com técnicas de simulação se mostrou eficiente, pois forneceu uma visão geral de forma contínua ao longo do processo de ensinoaprendizagem de Redes de Computadores. Com isso, pôde-se observar de forma mais 
V Congresso Brasileiro de Informática na Educação (CBIE 2016)

Anais do XXVII Simpósio Brasileiro de Informática na Educação (SBIE 2016)

precisa, como os estilos de aprendizagem sofrem alterações no decorrer do processo de ensino-aprendizagem. Desse modo, é possível identificar qual conteúdo é mais apropriado as necessidades individuais do aluno no momento certo, o que é um elemento fundamental para ambientes $U$-learning .

Esse trabalho apresentou resultados positivos com um grau mais preciso se comparado a outros que utilizam instrumentos de coleta de dados determinar o perfil do estudante. Tal abordagem foi realizada com baixo custo computacional proporcionado pela tecnologia de Cloud Computing, dado que foi reutilizado o hardware existente no laboratório de pesquisa GRECA/UFSM. Portanto, observou-se que as informações obtidas nesse trabalho podem apoiar a construção de ambientes adaptativos como o laboratório virtual U-Lab Cloud. Este ambiente se adapta ao contexto computacional do estudante, visto que verifica a velocidade de conexão, o tipo de dispositivo e o estilo cognitivo do aluno.

\section{Referências}

Bai, Y.; Shen, S.; Chen, L.; Zhuon, Y. Cloud learning: A new style. In: IEEE International Conference on Multimedia Technology (ICMT), 2011. Anais... IEEE, p. 3460-3463, 2011.

Caminero, A. et al. Obtaining university practical competences in engineering by means of virtualization and cloud computing technologies. In: IEEE International Conference on Teaching, Assessment and Learning for Engineerring (TALE), 2013. Anais... IEEE, 2013. p. 301-306.

Dey, A. K. Understanding and Using Context. Personal and Ubiquitous Computing, [S.1.], v.5, n.1, p.4-7, 2001.

Doelitzscher F. et al. Private cloud for collaboration and e-Learning services: From IaaS to SaaS. Journal Computing, [S.1.], v.91, p. 23-42, 2011.

Dorça, F. A. Uma abordagem estocástica baseada em Aprendizagem por Reforço para modelagem automática e dinâmica de Estilos de Aprendizagem de Estudantes em Sistemas Adaptativos e Inteligentes para Educação a Distância. 2012. Tese. (Doutorado em Ciências) - Faculdade de Engenharia Elétrica da Universidade Federal de Uberlândia.

Fillipetti, M. A. Uma arquitetura para a construção de laboratórios híbridos de redes de computadores remotamente acessíveis. 2008. Dissertação (Mestrado em Engenharia da Computação) - Instituto Pesquisas Tecnológicas do Estado de São Paulo (IPT).

Herpich, F.; Nunes, F. B.; Voss, G. B.; Medina, R. D. (2016). Three-Dimensional Virtual Environment and NPC: A Perspective about Intelligent Agents Ubiquitous. In F. M. Neto, R. de Souza, \& A. S. Gomes (Eds.), Handbook of Research on 3-D Virtual Environments and Hypermedia for Ubiquitous Learning (pp. 510-536). Hershey PA, USA: IGI Global.

Hooft, M. What is ubiquitous computing? In: Hooft and Swan, Ubiquitous Computing in Education Invisible technology, visible impact, London: Lawrence Erbaum Ass., p.3-17, 2006.

Iglesias, A. Learning teaching strategies in an adaptive and intelligent educational system through reinforcement learning. International Journal Applied Intelligence, [S.1.], v.31, n.1, p. 89-106, 2009.

Jadela Y.; Modi K. Cloud computing - concepts, architecture and challenges. In: IEEE International Conference on Computing, Electronics and Electrical Technologies (ICCEET). Kumaracoil, p. 877 - 880, 2012. 
V Congresso Brasileiro de Informática na Educação (CBIE 2016)

Anais do XXVII Simpósio Brasileiro de Informática na Educação (SBIE 2016)

Jeong, H-Y.; YI, G. A Service Based Adaptive U-Learning System Using UX. In: The Scientific Wordl Journal, [S.I.], 2014.

Mell, P.; GRANCE, T. The Nist Definition of Cloud Computing. In: National Institute of Standards and Technology, [S.1], p. 7, 2011.

Mozzaquatro, P. M. Adaptação do Mobile Learning Engine Moodle (MLE MOODLE) aos diferentes estilos cognitivos utilizando Hipermídia Adaptativa. 2010. Dissertação (Mestrado em Computação) Universidade Federal de Santa Maria.

Orgaz, G. B. et al. Clustering Avatars BehavioursFrom Virtual Worlds Interactions. In: International Workshop On Web Intelligence \& Communities - WI\&C'12, 4., New York, USA. Proceedings. ACM Press, p.1, 2012.

Piovesan, S. U-SEA: um ambiente de aprendizagem ubíquo utilizando cloud computing. 2011. Dissertação (Mestrado em Computação) - Universidade Federal de Santa Maria.

Rabello S. et al. Um Modelo para Colaboração em Ambientes Descentralizados de Educação Ubíqua. Rio de Janeiro, RJ: Anais... $23^{\circ}$ Simpósio Brasileiro de Informática na Educação (SBIE), v.23, n.1, 2012.

Voss, G. TCN ${ }^{s}$ - Teaching Computer Networks In a Free Immersive Virtual Environment. 2014. Dissertação (Mestrado em Computação) - Universidade Federal de Santa Maria.

Yahya, S.; Ahmad, E. A.; Jalil, K. A.; Mara, U. T. The definition and characteristics of ubiquitous learning : A discussion. International Journal of Education and Development using Information and Communication Technology, v. 6, p. 1-12, 2010.

Wang X.; Hembroff C. G.; Yedica R. Using VMware VCenter lab manager in undergraduate education for system administration and network security. In: Proc. of The 11th acm Conferene on Information Technology Education (SIGITE), (Midland, USA), 2010. 